\title{
Comparison of Canal Transportation and Centering Ability of ProGlider and WaveOne Gold Glider in Curved Canals
}

\author{
Gabriela Behne Miró ${ }^{1} \quad$ Flávia Sens Fagundes Tomazinho ${ }^{1}$ Edson Pelisser ${ }^{1}$ \\ Mariana Maciel Batista Borges ${ }^{2}$ Marco Antônio Hungaro Duarte ${ }^{2}$ Rodrigo Ricci Vivan² \\ Flares Baratto-Filho ${ }^{1,3}$
}

${ }^{1}$ School of Health Sciences, Universidade Positivo, Curitiba,

Paraná, Brazil

2Department of Dentistry, Endodontics and Dental Materials, Bauru Dental School, Universidade de São Paulo, Bauru, São Paulo, Brazil

${ }^{3}$ Department of Dentistry, Universidade da Região de Joinville,

Joinville, Santa Catarina, Brazil Address for correspondence Flávia Sens Fagundes Tomazinho,
DDS, PhD, School of Health Sciences, Universidade Positivo,
Rua Prof. Pedro Viriato Parigot de Souza, 5300, Curitiba,
Paraná 81280-330, Brazil (e-mail: flavia.tomazinho@gmail.com).

Eur J Dent:2020;14:639-643

\begin{abstract}
Keywords

- canal transportation

- centralization

- endodontic

- ProGlider

- WaveOne Gold Glider

Objectives The aim of this study was to compare the volume variation and maintenance of the root canal position when using the ProGlider 16.02 (PG) and the WaveOne Gold Glider 15.02 (WOGG) file systems for glide path preparation.

Materials and Methods Twenty-four moderately curved mesiobuccal canals of maxillary first molars were selected and randomly divided into two groups: PG and WOGG. The selected teeth were scanned using microtomography before and after root canal preparation to assess centralization and linear transport at 1, 3, 5, and $7 \mathrm{~mm}$ from the apical foramen.

Statistical Analysis The data were analyzed with the Mann-Whitney U test. The level of significance was $5 \%$.

Results There were no significant differences in volume variation or root canal transport $(p>0.05)$. There was a significant difference in the centralization of the root canal at $3 \mathrm{~mm}$ from the foramen $(p<0.05)$.

Conclusions WOGG and PG instruments presented similar results regarding the root canal volume increase and transport. WOGG caused higher decentralization at $3 \mathrm{~mm}$ from the apical foramen.
\end{abstract}

\section{Introduction}

The endodontic glide path is used for the exploration and pre-enlargement of the root canal to minimize errors in biomechanical preparation, such as canal transport and deviations. ${ }^{1-3}$ This procedure can be conducted with instruments made of nickel-titanium (NiTi) alloys with different heat treatments, designs, and conicities. Pre-enlargement provided by the glide path allows the root canal preparation instruments to work with less torsional stress, reducing the risk of instrument fracture. ${ }^{1,3-9}$
Although not statistically significant, the highest mean transportation values have been observed when the glide path is not prepared before the final canal instrumentation. ${ }^{1}$ Therefore, the performance of the file system might be enhanced by preparation of a glide path before the final shaping. ${ }^{10,11}$

Previous studies ${ }^{12,13}$ have compared the effectiveness of glide path instruments manufactured with various NiTi alloys and recommended the use of M-Wire NiTi alloy instruments. These instruments obtain a more predictable patency, especially in curved canals. ${ }^{14}$ Among these instruments is the 
ProGlider (PG) (Dentsply Sirona; Ballaigues, Switzerland), which is used in a continuous rotary motion. The PG is manufactured using M-Wire NiTi alloy, has a quadrangular cross section with an initial diameter of $0.16 \mathrm{~mm}$, and a progressive taper ranging from 2 to $8 \%{ }^{12}$ The WaveOne Gold Glider (WOGG) (Dentsply Sirona) is used in a reciprocating motion and is another option for glide path preparation. The WOGG is manufactured using NiTi Gold alloy, has a parallelogram cross-section, along with a semi-active guiding tip $0.15 \mathrm{~mm}$ in diameter, and variable conicity ranging from 2 to $6 \%{ }^{9}$

The progressive tapered design of these file systems provides a glide path and preliminary enlargement of the root canal in the middle and coronal regions. This feature has been reported to reduce stress to the subsequent shaping NiTi rotary instruments. ${ }^{15}$ However, little data exists on the capacity of these single-file glide path techniques to maintain the original root canal anatomy. This study aims to elucidate the role of these file systems in glide path preparation and preserving the root canal integrity during canal instrumentation. This might help clinicians in selecting the most favorable instrument system for root canal preparation.

Previous studies have evaluated the root canal transport and centralization when the WOGG was used for the glide path preparation. ${ }^{9,16}$ Another study compared the volume, transport, and centralization between the PG and the WOGG in the mesial canals of lower first molars. ${ }^{16}$ The objective of our study was to compare the volume variation and the root canal shape maintenance capacity using the PG and the WOGG in the mesiobuccal canals of maxillary molars. The null hypothesis of this study states the following: (1) there is no difference in the root canal volume variation between the PG and the WOGG file systems, and (2) there is no difference in the root canal position maintenance between the two systems.

\section{Materials and Methods}

This study was approved by the Institutional Ethics Committee (2.441.608).

\section{Specimen Selection}

The mesiobuccal canals of 24 human permanent maxillary first molars were used in this study. The sample size (n) was based on the previous studies published in the literature. ${ }^{2,9,17}$ The teeth had been previously radiographed and were selected based on the presentation criteria of curvature between 10 and 30 degrees in the buccal roots, as determined by the Schneider method. ${ }^{18}$ In the interest of the research, teeth with curvature greater than 30 or less than 10 degrees, incomplete root formation, internal or external resorption, previous endodontic treatment, and decayed tissue were discarded. The selected teeth had an average length of $20 \mathrm{~mm}$ and were stored in water throughout the experimental phase.

\section{Specimen Preparation}

After access cavity preparation, the mesiobuccal canals were explored with a \#10 K file (Dentsply Sirona). The root canal was explored up to $1 \mathrm{~mm}$ beyond the root apex to confirm foraminal patency. ${ }^{9,14,19,20}$ The teeth were then randomly divided into two experimental groups: PG (glide path with Pro Glider 16.02 instrument; $n=12$ ) and WOGG (glide path with WaveOne Gold Glider 15.02 instrument; $n=12$ ). The actual length of each canal was determined by microscopic visualization of the tip of the \#10 K file in the foramen, and the standardized working length $(\mathrm{WL})$ was $1 \mathrm{~mm}$ shorter than the actual canal length.

All teeth were prepared by a single operator using the electric motor X-Smart Plus (Dentsply Sirona). The PG instrument was used at a speed of $300 \mathrm{rpm}$ and $3 \mathrm{~N}-\mathrm{cm}$ torque, ${ }^{21}$ and the WOGG was used in the WaveOne mode. All instruments were used only once. The root canal was irrigated with $2 \mathrm{~mL}$ of $2.5 \%$ sodium hypochlorite solution at each instrument insertion and was cleaned after each introduction until the WL was reached. Final irrigation was performed with $5 \mathrm{~mL}$ of $17 \%$ ethylenediaminetetraacetic acid for 3 minutes followed by $5 \mathrm{~mL}$ of distilled water.

\section{Computed Microtomography, Reconstruction, and Evaluation of Images}

Evaluation of the volume variation and root canal position maintenance capacity was performed using images obtained by computed microtomography (micro-CT). Teeth were scanned with a microtomograph (SkyScan 1174v2; Bruker-microCT, Kontich, Belgium) before and after the root canal preparation. Image acquisition parameters were $50 \mathrm{kV}$, $800 \mu \mathrm{A}$, and an isotropic voxel of $16.8 \mu \mathrm{m}$. The reconstruction of the images in tomographic sections was performed with the aid of the NRecon (SkyScan; Kontich, Belgium) program. The realignment of the pre and postinstrumentation images was then performed using the DataViewer (SkyScan) program. The computed tomography analyzer (CTAn; SkyScan, Kontich, Belgium) software was used to compare two-dimensional slices obtained before and after instrumentation. It allowed direct visualization of the location, as well as the amount and direction of apical transport. The four sections of interest were $1,3,5$, and $7 \mathrm{~mm}$ from the apical foramen. The sections were determined by identifying the slice in which the foramen was first visible ( $0 \mathrm{~mm}$ mark) and then adding 20 slices to reach $1 \mathrm{~mm}, 40$ slices to reach $3 \mathrm{~mm}$, with continuation of adding slices until $7 \mathrm{~mm}$ was reached. The same slices were compared before and after instrumentation.

\section{Root Canal Volume Assessment}

The root canal volume was measured before and after the use of the glide path instruments. Volume variation was determined by subtracting the volume of the canal before the glide path preparation from the canal volume after the glide path preparation using the following formula ${ }^{22}$ :

(V2-V1) for variation, and

$((V 2 / V 1)-1) \times 100$ for percentage

$V 1$ is the volume before the glide path preparation, and $V 2$ is the volume after the glide path preparation. 


\section{Evaluation of Root Canal Transport and Centralization}

The method developed by Gambill et $\mathrm{a}^{17}$ was used to determine the degree of linear transport ( - Fig. 1). Pre and postinstrumentation measurements were compared with assess the magnitude and direction of apical transport using the following formula:

$$
(a 1-a 2)-(b 1-b 2),
$$

where $a 1$ is the shortest distance between the mesial portions of the root and uninstrumented canal, $a 2$ is the shortest distance between the mesial portions of the root and instrumented canal, $b 1$ is the shortest distance between the distal portions of the root and uninstrumented canal, and $b 2$ is the shortest distance between the distal portions of the root and instrumented canal. Result " 0 " indicates no canal transport, a positive value represents movement to the outer surface, and a negative value represents movement to the inner surface or toward the furcation.

To calculate the centralization, ${ }^{17}$ we used the same measures used for the linear transport calculation; however, the applied formula was as follows:

$$
(a 1-a 2) /(b 1-b 2) \text { or }(b 1-b 2) /(a 1-a 2) \text {. }
$$

If different values were obtained from the two applied formulas, the lower value of the obtained measures was used for the result. Results equal to "1" indicated perfect centralization, while " 0 " represented complete decentralization.

\section{Statistical Analysis}

Preliminary analysis of data normality was performed with the Kolmogorov-Smirnov test showing non-normal distribution. According to these criteria, the Mann-Whitney $U$ test was used to compare the samples. The GraphPad Prism 6 software (GraphPad Software Inc.; La Jolla, San Diego, California, United States) was used for all statistical analysis, with a significance level of $5 \%(p<0.05)$.

\section{Results}

- Table 1 shows the median, minimum, and maximum values of volume variation and the percentage of volume variation. Glide path preparation with the PG and the WOGG instruments was compared, and there were no statistically significant differences between the groups $(p>0.05)$.

- Table 2 shows the median, minimum, and maximum values of linear transport, and the canal centralization (centering ratios) at the four levels evaluated, comparing the glide path PG and WOGG instruments.

There was no statistically significant difference between the groups in the linear root canal transport $(p>0.05)$. There was a statistically significant difference between the instruments in the root canal centralization at $3 \mathrm{~mm}$ from the apical foramen $(p<0.05)$. In the other regions examined, there were no differences between the instruments.

\section{Discussion}

There are limited studies in literature comparing the glide path instruments, such as the PG and the WOGG; thus, further research is needed. Our study evaluated the volume variation and the root canal position maintenance capacity when using the PG and the WOGG instruments for glide path

Table 1 Median, minimum, and maximum values of volume variation in $\mathrm{mm}^{3}$ and percentage of volume increase

\begin{tabular}{|l|l|l|l|}
\hline & PG & WOGG & $p$-Value \\
\hline$\Delta$ volume & 0.105 & 0.105 & 0.976 \\
\hline$\left(\mathrm{mm}^{3}\right)$ & $(0.010-0.500)$ & $(0.000-0.220)$ & \\
\hline$\Delta$ volume & 8.295 & 9.415 & 0.707 \\
\hline$(\%)$ & $(0.980-33.54)$ & $(0.800-33.36)$ & \\
\hline
\end{tabular}

Abbreviations: PG, ProGlider; WOGG, WaveOne Gold Glider. Mann-Whitney U test $(p<0.05)$.
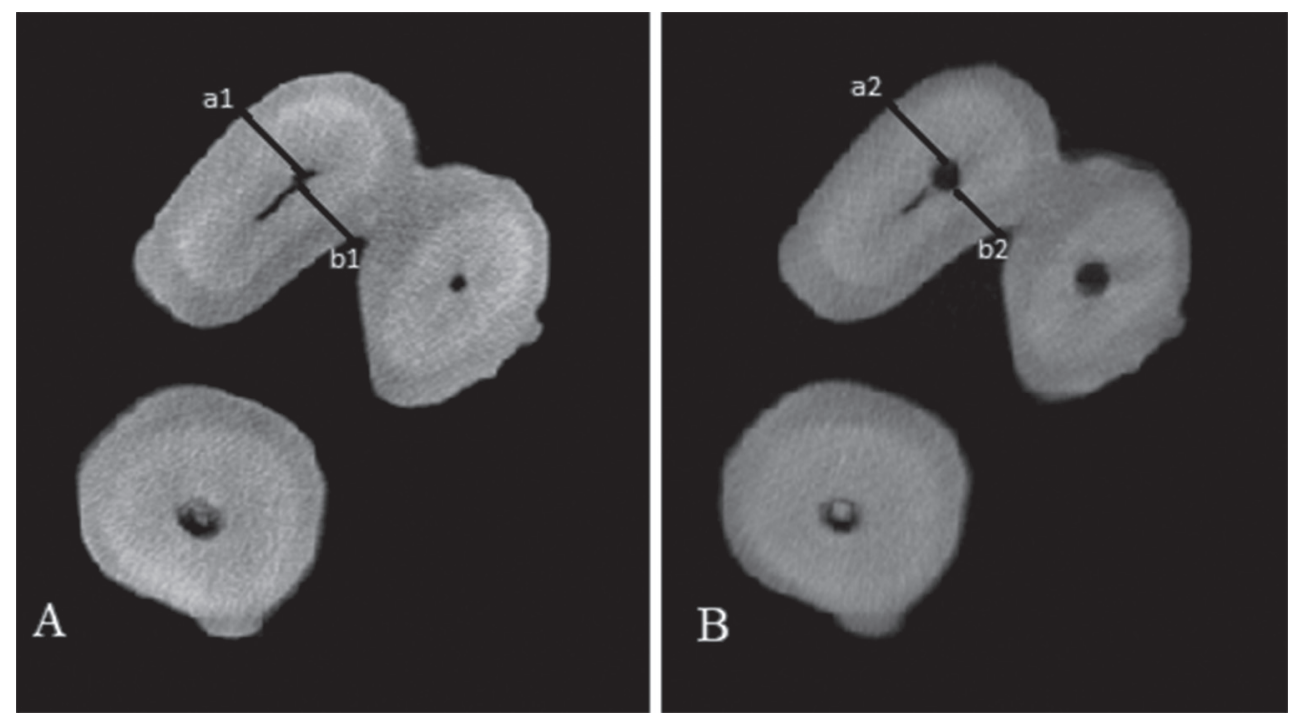

Fig. 1 Lines drawn on the same tooth at preinstrumentation (A) in the mesial (a1) and distal (b1) walls, and at postinstrumentation (B) in the mesial (a2) and distal (b2) walls. 
Table 2 Median, minimum, and maximum values of transportation, and centering ratios values (in mm) at 1, 3, 5, and $7 \mathrm{~mm}$ from the apical foramen comparing the glide path instruments (PG and WOGG)

\begin{tabular}{|l|l|l|l|l|l|l|l|}
\hline \multirow{2}{*}{ Level } & Assessment & \multicolumn{3}{|c|}{ PG } & \multicolumn{3}{c|}{ WOGG } \\
\cline { 3 - 8 } & & Med & Min & Max & Med & Min & Max \\
\hline $1 \mathrm{~mm}$ & Transportation & $0.002^{\mathrm{a}}$ & 0.112 & 0.120 & $0.000^{\mathrm{a}}$ & 0.118 & 0.112 \\
\hline & Centering ratio & $0.059^{\mathrm{a}}$ & 0.000 & 0.954 & $0.000^{\mathrm{a}}$ & 0.000 & 0.939 \\
\hline $3 \mathrm{~mm}$ & Transportation & $0.001^{\mathrm{a}}$ & 0.077 & 0.032 & $-0.004^{\mathrm{a}}$ & 0.077 & 0.124 \\
\hline $5 \mathrm{~mm}$ & Centering ratio & $0.598^{\mathrm{a}}$ & 0.000 & 0.991 & $0.000^{\mathrm{b}}$ & 0.000 & 0.636 \\
\hline & Transportation & $0.041^{\mathrm{a}}$ & 0.178 & 0.067 & $0.000^{\mathrm{a}}$ & 0.077 & 0.179 \\
\hline $7 \mathrm{~mm}$ & Centering ratio & $0.000^{\mathrm{a}}$ & 0.000 & 0.982 & $0.182^{\mathrm{a}}$ & 0.000 & 1.000 \\
\hline & Transportation & $0.064^{\mathrm{a}}$ & 0.227 & 0.074 & $-0.012^{\mathrm{a}}$ & 0.168 & 0.114 \\
\hline
\end{tabular}

Abbreviations: PG, ProGlider; WOGG, WaveOne Gold Glider.

In the evaluation of transportation and centering ratios, superscripted letters indicate significant differences between the groups. Mann-Whitney $U$ test $(p<0.05)$.

preparation. The main differences between these instruments are the kinematics employed and the NiTi alloy used.

The transportation after the root canal shaping procedures, the noncentral root canal preparation, and insufficient or excessive instrumentation of the tooth structure may have negative effects on the prognosis. ${ }^{5}$ Therefore, assessing the quality of root canal preparation is of significant importance for selecting the appropriate file system. ${ }^{16}$ A reduced number of failures related to instrumentation ${ }^{5}$ and a reduction in preparation time have contributed to the growing popularity and clinical acceptability of the glide path instruments. ${ }^{23}$ Compared with manual glide path preparation, mechanical glide path preparation seems to be less technique-sensitive, resulting in an improved preservation of the canal anatomy, fewer canal aberrations, reduced time required for shaping, and a lower incidence of postoperative pain.., 5

To investigate the two single-file glide path preparation systems, we used moderately curved mesiobuccal root canals of maxillary molars. The pre- and postprocedure images of the same sample were combined by means of advanced matching and comparison features during micro-CT analysis to obtain fully measurable images., ${ }^{3,16}$ The micro-CT method has been accepted as an important scientific instrument for assessing the efficiency of shaping methods of various file systems, and its repeatability and accuracy have been proven in several studies. $2,5,19,24,25$

In this study, four levels were chosen for the analysis of root canal transport and centralization: $1,3,5$, and $7 \mathrm{~mm}$ from the foramen. ${ }^{26}$ These measurements represent the foraminal, apical, middle, and coronal third segments of the root, which usually have canal curvatures resulting in a high vulnerability to iatrogenic accidents. The crowns were kept intact to reproduce the clinical situation in which the cervical dentin interference could produce tension or resistance during root canal instrumentation. ${ }^{22}$

The null hypotheses of this study were as follows: (1) Not rejected because there was no difference in the volume variation between the use of the PG and the WOGG. (2) Rejected because there was a difference in the root canal centralization when the PG and the WOGG instruments were compared.

In this study, no statistically significant difference was observed between the groups regarding the canal volume variation. The instruments examined have similar diameter and conicities; however, the differences in kinematics of use, properties of the alloy used, and cross section did not result in differences in the dentin removal capacity. Unlike the results found in this study, a study by Aydin et al, ${ }^{16}$ which also used micro-CT analysis, reported that the PG showed a significant volume increase when compared with the WOGG and the R-Pilot (VDW, Munich, Germany). However, the study conducted by van der Vyver et al ${ }^{19}$ corroborates the results found in our study. Other studies that evaluated the PG showed that this instrument performed well, had greater centralization, and reduced transport compared with hand instruments, ${ }^{7,14,19}$ thus, increasing the safety in canal preparation. ${ }^{7,14,19,20,27}$ The properties of the M-Wire NiTi alloy and the taper of the instruments allow for favorable results when compared with the conventional NiTi alloy. ${ }^{9,14}$

In contrast to the present study, the study conducted by Aydin et $\mathrm{al}^{16}$ showed higher transport and lower centralization with the PG when compared with the WOGG and the R-Pilot in different sections of the root canal. In our study, the WOGG resulted in greater decentralization at $3 \mathrm{~mm}$ from the apical foramen. This may be because of the file movement, NiTi alloy, or the methodology used. In addition to the results found in this study, other studies have shown the WOGG instrument helps maintain the root canal position., ${ }^{9,16}$

Within the limitations of the present study, it can be concluded that the glide path instruments, the PG and the WOGG, presented similar results in relation to the increase in root canal volume and transportation. However, the WOGG instrument resulted in higher decentralization at the $3 \mathrm{~mm}$ portion. 


\section{Conflict of Interest}

None declared.

\section{References}

1 Elnaghy AM, Elsaka SE. Evaluation of root canal transportation, centering ratio, and remaining dentin thickness associated with ProTaper Next instruments with and without glide path. J Endod 2014;40(12):2053-2056

2 Jatahy Ferreira do Amaral RO, Leonardi DP, Gabardo MC, Coelho BS, Oliveira KV, Baratto Filho F. Influence of cervical and apical enlargement associated with the WaveOne system on the transportation and centralization of endodontic preparations. J Endod 2016;42(4):626-631

3 Kirchhoff AL, Chu R, Mello I. Garzon AD, dos Santos M, Cunha RS. Glide path management with single- and multiple-instrument rotary systems in curved canals: a micro-computed tomographic study. J Endod 2015;41(11):1880-1883

4 Berutti E, Cantatore G, Castellucci A, et al. Use of nickeltitanium rotary PathFile to create the glide path: comparison with manual preflaring in simulated root canals. J Endod 2009;35(3):408-412

5 Pasqualini D, Bianchi CC, Paolino DS, et al. Computed micro-tomographic evaluation of glide path with nickel-titanium rotary PathFile in maxillary first molars curved canals. J Endod 2012;38(3):389-393

6 De-Deus G, Arruda TE, Souza EM, et al. The ability of the Reciproc R25 instrument to reach the full root canal working length without a glide path. Int Endod J 2013;46(10):993-998

7 Paleker F, van der Vyver PJ. Comparison of canal transportation and centering ability of K-files, ProGlider File, and G-Files: a micro-computed tomography study of curved root canals. J Endod 2016;42(7):1105-1109

8 Maniglia-Ferreira C, de Almeida Gomes F, Ximenes T, et al. Influence of reuse and cervical preflaring on the fracture strength of reciprocating instruments. Eur J Dent 2017;11(1): 41-47

9 Vorster M, van der Vyver PJ, Paleker F. Canal transportation and centering ability of WaveOne Gold in combination with and without different glide path techniques. J Endod 2018;44(9): 1430-1435

10 de Carvalho GM, Sponchiado Junior EC, Garrido AD, Lia RC, Garcia LdaF, Marques AA. Apical transportation, centering ability, and cleaning effectiveness of reciprocating single-file system associated with different glide path techniques. J Endod 2015;41(12):2045-2049

11 Troiano G, Dioguardi M, Cocco A, Zhurakivska K, Ciavarella D, Muzio LL. Increase in [corrected] the glyde path diameter improves the centering ability of F6 Skytaper. [published correction appears in Eur J Dent. 2018 Jul-Sep;12(3): 467] Eur J Dent 2018;12(1):89-93

12 Al-Sudani D, Plotino G, Grande N, Rengo S, Simeone M. Cyclic fatigue of glide path rotary NiTi files in a double (S-Shaped) simulated curvature. Dentistry 2016;6:1-3
13 Capar ID, Kaval ME, Ertas H, Sen BH. Comparison of the cyclic fatigue resistance of 5 different rotary pathfinding instruments made of conventional nickel-titanium wire, M-wire, and controlled memory wire. J Endod 2015;41(4):535-538

14 Alovisi M, Cemenasco A, Mancini L, et al. Micro-CT evaluation of several glide path techniques and ProTaper Next shaping outcomes in maxillary first molar curved canals. Int Endod J 2017;50(4):387-397

15 Berutti E, Paolino DS, Chiandussi G, et al. Root canal anatomy preservation of WaveOne reciprocating files with or without glide path. J Endod 2012;38(1):101-104

16 Aydın ZU, Keskin NB, Özyürek T, Geneci F, Ocak M, Çelik HH. Microcomputed assessment of transportation, centering ratio, canal area, and volume increase after single-file rotary and reciprocating glide path instrumentation in curved root canals: a laboratory study. J Endod 2019;45(6):791-796

17 Gambill JM, Alder M, del Rio CE. Comparison of nickel-titanium and stainless steel hand-file instrumentation using computed tomography. J Endod 1996;22(7):369-375

18 SchneiderSW.Acomparison of canal preparationsinstraightand curved root canals. Oral Surg Oral Med Oral Pathol 1971;32(2): 271-275

19 van der Vyver PJ, Paleker F, Vorster M, de Wet FA. Microcomputed tomographic evaluation of two single rotary glide path systems. Int Endod J 2019;52(3):352-358

20 van der Vyver PJ, Paleker F, Vorster M, de Wet FA. Root canal shaping using nickel titanium, M-Wire, and Gold Wire: a micro-computed tomographic comparative study of One Shape, ProTaper Next, and WaveOne Gold instruments in maxillary first molars. J Endod 2019;45(1):62-67

21 Topçuoğlu HS, Topçuoğlu G, Düzgün S. Resistance to cyclic fatigue of PathFile, ScoutRaCe and ProGlider glide path files in an S-shaped canal. Int Endod J 2018;51(5):509-514

22 Hashem AA, Ghoneim AG, Lutfy RA, Foda MY, Omar GA. Geometric analysis of root canals prepared by four rotary NiTi shaping systems. J Endod 2012;38(7):996-1000

23 Vorster M, van der Vyver PJ, Paleker F. Influence of glide path preparation on the canal shaping times of WaveOne Gold in curved mandibular molar canals. J Endod 2018;44(5):853-855

24 Gergi R, Osta N, Bourbouze G, Zgheib C, Arbab-Chirani R, Naaman A. Effects of three nickel titanium instrument systems on root canal geometry assessed by micro-computed tomography. Int Endod J 2015;48(2):162-170

25 Elashiry MM, Saber SE, Elashry SH. Comparison of shaping ability of different single-file systems using microcomputed tomography. Eur J Dent 2020;14(1):70-76

26 Arruda EDS, Sponchiado-Júnior EC, Pandolfo MT, Fredson MAC, Roberi Garcia LDF, Marques AA. Apical transportation and centering ability after root canal filling removal using reciprocating and continuous rotary systems: a CBCT study. Eur J Dent 2019;13(4):613-618

27 Zheng L, Ji X, Li C, Zuo L, Wei X. Comparison of glide paths created with K-files, PathFiles, and the ProGlider file, and their effects on subsequent WaveOne preparation in curved canals. BMC Oral Health 2018;18(1):152-157 